\title{
Kernel Based Fuzzy Ant Clustering with Partition validity
}

\author{
Yuhua Gu and Lawrence O. Hall
}

\begin{abstract}
We introduce a new swarm intelligence based algorithm for data clustering with a kernel-induced distance metric. Previously a swarm based approach using artificial ants to optimize the fuzzy c-means (FCM) criterion using the Euclidean distance was developed. However, FCM is not suitable for clusters which are not hyper-spherical and FCM requires the number of cluster centers be known in advance. The swarm based algorithm determines the number of cluster centers of the input data by using a modification to the fuzzy cluster validity metric proposed by Xie and Beni. The partition validity metric was developed based on the kernelized distance measure. Experiments were done with three data sets; the Iris data, an artificially generated data set and a Magnetic Resonance brain image data set. The results show how effectively the kernelized version of validity metric with a fuzzy ant method finds the number of clusters in the data and that it can be used to partition the data.
\end{abstract}

\section{INTRODUCTION}

Many clustering algorithms developed using the principals of Swarm Intelligence are distributed, flexible and robust. The algorithms simulate the co-operative and stochastic iterative behavior of a colony of ants. The goal of clustering is to separate a dataset into self-similar groups such that the examples in the same group have more similarity than the examples in other groups. There is a drawback to clustering algorithms such as fuzzy c-means (FCM) which require prior knowledge about the number of clusters in the data, which may not be known for new data. In previous work [2], a swarm based fuzzy clustering algorithm using the Xie-Beni [1] partition validity metric was presented, which determined the number of clusters very well for several data sets. The Xie-Beni validity metric used in [2] was based on the fuzzy c-means clustering algorithm, which was modified so that a membership matrix does not need to be computed [3]. A threshold on the percentage of examples was applied to cluster size to eliminate very small clusters or empty clusters, as the FCM functional has a strong bias towards equal size clusters.

The FCM algorithm often uses a Euclidean distance measure, which is fine for most noise-free data. However difficulties arise when data is corrupted by noise or outliers. In order to deal with these kinds of data sets, kernel methods have been widely applied. The fundamental idea of kernel methods is to transform the original low-dimensional input space into a higher dimensional feature space through some (nonlinear) mapping, therefore some complex linear problems in the original space can possibly be solved in the transformed highdimensional space. The kernelized FCM (KFCM) [4] used

Yuhua Gu and L. O. Hall are with Dept. of Computer Science \& Engineering University of South Florida, Tampa FL 33620 U.S.A.

$<$ hall, yuhua $>$ @csee.usf.edu a kernel function as a substitute for the inner product in the original space, which is like mapping the space into higherdimensional feature space. The objective function for FCM then has a new form.

There have been a number of other approaches to incorporating kernels into fuzzy clustering algorithms. These include enhancing clustering algorithms designed to handle different shape clusters [8]. An approach which is designed for incomplete data [6] and a different formulation of a centroid-based clustering algorithm [5]. In [7] a Mercer kernel-induced clustering method was described. The clustering was performed in the transformed feature space after a nonlinear data transformation. A drawback was that the clustering result was difficult to exactly represent due to the fact that cluster centers may not have a pre-image in the original data space.

Since the Xie and Beni criterion is founded on the FCM algorithm, a new Xie-Beni partition validity metric (kernelbased Xie-Beni criterion) was reformulated based on the kernel method from [4]. This required a new objective function without membership matrices [3]. We will show the validity metric with a fuzzy ant method can pick the right number of clusters in the data.

The outline of the paper is as follows: Section 2 briefly describes the KFCM clustering algorithm and the new XieBeni Partition validity metric. In Section 3, we discuss ant based clustering using partition validity to evaluate the goodness of partitions. Section 4 explains the data sets used and the experimental results. A summary and discussion is given in Section 5.

\section{KERNEL-BASED FUZZY CLUSTERING AND CORRESPONDING PARTITION VALIDITY}

In [3] the authors proposed a reformulation of the optimization criteria used in a couple of common clustering objective functions. The FCM objective function which is minimized by the original clustering algorithm, often using the Euclidean distance measure, is shown in (1).

$$
\begin{gathered}
J_{m}(U, \beta)=\sum_{i=1}^{c} \sum_{k=1}^{n} U_{i k}^{m} D_{i k}\left(x_{k}, \beta_{i}\right) \\
U_{i k}=\frac{D_{i k}\left(x_{k}, \beta_{i}\right)^{\frac{1}{1-m}}}{\sum_{j=1}^{c} D_{j k}\left(x_{k}, \beta_{j}\right)^{\frac{1}{1-m}}}
\end{gathered}
$$

where

$U_{i k}$ : Membership of the $k^{t h}$ object in the $i^{\text {th }}$ cluster

$\beta_{i}$ : The $i^{\text {th }}$ cluster prototype

$m>1$ : The degree of fuzzification

$c \geq 2$ : Number of clusters 
$\mathrm{n}$ : Number of data points

$D_{i k}\left(x_{k}, \beta_{i}\right)$ : Distance of $x_{k}$ from the $i^{t h}$ cluster center

In [4], the author proposed a KFCM algorithm and modified its objective function with the mapping $\phi$ as follows:

$$
J_{m}^{\phi}=\sum_{i=1}^{c} \sum_{k=1}^{n} U_{i k}^{m}\left\|\phi\left(x_{k}\right)-\phi\left(\beta_{i}\right)\right\|^{2}
$$

where $D_{i k}$ in (1) was replaced by $\left\|\phi\left(x_{k}\right)-\phi\left(\beta_{i}\right)\right\|^{2}$ and $\phi$ is a nonlinear transformation into a higher (possibly infinite)dimensional feature space $\mathrm{F}$. Then the inner product between $\phi(x)$ and $\phi(y)$ in the feature space $\mathrm{F}$ is : $\phi(x)^{T} \phi(y)=$ $K(x, y)$

The radial basis function (4) is one possibility:

$$
K(x, y)=\exp \left(\frac{-\left(\sum_{i=1}^{d}\left|x_{i}-y_{i}\right|^{a}\right)^{b}}{\sigma^{2}}\right)
$$

Since we used the typical radial basis function kernel (4), and the kernel substitution $K(x, x)=1$ for all $\mathrm{x}$, we have

$$
\begin{aligned}
D_{i k}\left(x_{k}, \beta_{i}\right) & =\left\|\phi\left(x_{k}\right)-\phi\left(\beta_{i}\right)\right\|^{2} \\
& =\phi\left(x_{k}\right)^{T} \phi\left(x_{k}\right)+\phi\left(\beta_{i}\right)^{T} \phi\left(\beta_{i}\right)-2 \phi\left(x_{k}\right)^{T} \phi\left(\beta_{i}\right) \\
& =K\left(x_{k}, x_{k}\right)+K\left(\beta_{i}, \beta_{i}\right)-2 K\left(x_{k}, \beta_{i}\right) \\
& =2-2 K\left(x_{k}, \beta_{i}\right)
\end{aligned}
$$

The $\mathrm{U}$ matrix for the reformulated criterion is denoted as $U^{\phi}$ (6), which can also be easily computed by substituting (5) into the original version (2).

$$
U_{i k}^{\phi}=\frac{\left[1-K\left(x_{k}, \beta_{i}\right)\right]^{\frac{1}{1-m}}}{\sum_{j=1}^{c}\left[1-K\left(x_{k}, \beta_{j}\right)\right]^{\frac{1}{1-m}}}
$$

The corresponding reformulated objective function $R_{m}^{\phi}$ is obtained by substituting (5) and (6) into (3):

$$
\begin{aligned}
R_{m}^{\phi}(\beta, X)= & \sum_{i=1}^{c} \sum_{k=1}^{n}\left\{\frac{\left[1-K\left(x_{k}, \beta_{i}\right)\right]^{\frac{1}{1-m}}}{\sum_{j=1}^{c}\left[1-K\left(x_{k}, \beta_{j}\right)\right]^{\frac{1}{1-m}}}\right\}^{m} \\
& *\left[2-2 K\left(x_{k}, \beta_{i}\right)\right] \\
= & 2 * \sum_{i=1}^{c} \sum_{k=1}^{n} \frac{\left[1-K\left(x_{k}, \beta_{i}\right)\right]^{\frac{m}{1-m}}}{\left\{\sum_{j=1}^{c}\left[1-K\left(x_{k}, \beta_{j}\right)\right]^{\frac{1}{1-m}}\right\}^{m}} \\
& *\left[1-K\left(x_{k}, \beta_{i}\right)\right] \\
= & 2 * \sum_{i=1}^{c} \sum_{k=1}^{n} \frac{\left[1-K\left(x_{k}, \beta_{i}\right)\right]^{\frac{1}{1-m}}}{\left\{\sum_{j=1}^{c}\left[1-K\left(x_{k}, \beta_{j}\right)\right]^{\frac{1}{1-m}}\right\}^{m}} \\
= & 2 * \sum_{k=1}^{n} \sum_{i=1}^{c} \frac{\left[1-K\left(x_{k}, \beta_{i}\right)\right]^{\frac{1}{1-m}}}{\left\{\sum_{j=1}^{c}\left[1-K\left(x_{k}, \beta_{j}\right)\right]^{\frac{1}{1-m}}\right\}^{m}} \\
= & 2 * \sum_{k=1}^{n} \frac{\sum_{i=1}^{c}\left[1-K\left(x_{k}, \beta_{i}\right)\right]^{\frac{1}{1-m}}}{\left\{\sum_{j=1}^{c}\left[1-K\left(x_{k}, \beta_{j}\right)\right]^{\frac{1}{1-m}}\right\}^{m}} \\
& (\text { substituting for } i) \\
= & 2 * \sum_{k=1}^{n}\left[\sum_{j=1}^{c}\left[1-K\left(x_{k}, \beta_{j}\right)\right]^{\frac{1}{1-m}}\right]^{1-m} \\
& \\
& \\
& \\
&
\end{aligned}
$$

The function $R_{m}^{\phi}$ depends only on the cluster prototypes and not on the $U^{\phi}$ matrix.

Now the kernel-based Xie-Beni partition validity metric is:

$$
X B(\beta, X)=\frac{R_{m}^{\phi}(\beta, X)}{n *\left[\min _{i \neq j} 2 *\left(1-K\left(\beta_{i}, \beta_{j}\right)\right)\right]}
$$

\section{FUZZY ANTS ALGORITHM}

Previous work on a Fuzzy ants clustering algorithm has been discussed in [2][10][11]. The approach here is along the same lines with a kernel distance metric reformulation. The ants co-ordinate to move cluster centers in feature space to search for optimal cluster centers. The input data is first normalized between 0 and 1 . Then a number of ants are assigned to each feature. Each ant belongs to a partition for a particular feature of a cluster. The ant never changes the feature, cluster or partition assigned to it. The goodness of the partition is evaluated by using the kernel Xie-Beni criterion (8) after an epoch. An epoch consists of randomly moving the cluster centers for a fixed number of iterations. Each ant has a memory ( 5 here). If the current partition is better than any of the previous partitions in the ant's memory, then this location is remembered by the ant, otherwise, the ant goes back to a better location or continues from the current location with a given probability. Since the ant memory is limited, this ensures a good partition is never replaced by a bad partition. The ants are able to move from 0 to 1 and from 1 to 0 two opposite directions in the feature space. If the ant 
happens to reach the end of the feature space during random movement, the ant reverses its direction. The movement of an ant can be a random distance between $D_{\min }$ and $D_{\max }$ (given parameters). It can also elect not to move for an epoch with a probability $P_{\text {rest }}$ or it has a probability of continuing $P_{\text {continue }}$ in the same direction as the ant was moving at the start of the epoch. At the end of an epoch, if the partition is not better than any of the old partitions in the ant memory, then with a probability $P_{\text {ContinueCurrent }}$ it continues with the current partition. Otherwise, with a probability 0.6 the ant chooses to go back to the best known partition, with a probability 0.2 the ant goes back to the second best known partition, with a probability 0.1 the ant goes to the third best known partition, with a probability 0.075 the ant goes to the fourth best known partition and with a probability 0.025 the ant goes to the worst known partition. After a fixed number of epochs the ants stop.

The search begins with more clusters than are expected in the data. The ant based method produces some empty clusters or some with few examples assigned to them which are possibly spurious. Nearly empty clusters indicate that there are less true clusters than our initial guess. The (nearly) empty clusters can be eliminated by choosing the right threshold value for each data set if we expect approximately equal sized clusters. The examples of the eliminated clusters would then be reassigned to the nearest centroid.

\section{EXPERIMENTS}

The first data set we used to evaluate the kernelized ant based algorithm was the well-known Iris data set. It has four continuous valued features, 150 examples, and three classes, 50 examples in each class. However, there is one class that is linearly separable from the other two classes and many partition metrics will report there are only two classes. The second data set had 2 continuous attributes, 5 classes and 1000 examples. It was generated using a Gaussian distribution, the classes are slightly unequally sized [11] $(248,132,217,192$ and 211 respectively). The third data set is a Magnetic Resonance brain image, which comes from a SIEMENS MRI machine at the University of Miami. This data set only contains one feature (T1-weighted image), the magnetic field strength is 3 Tesla, the data itself was corrupted by the intensity inhomogeneity induced by the radio-frequency coil in MRI. Therefore, a pre-processed step [9] was performed to correct the data. For this data set, a reasonable cluster number may be three, such as CSF (cerebro-spinal fluid), gray matter and white matter. The intensity image is 128 by 128 . With air pixels removed, the total number of voxels (examples) is 6535 .

\section{A. Experimental parameters}

The values of the parameters used in the fuzzy ant algorithm are shown in Table I. Notice that the parameters $\sigma$, a and $\mathrm{b}$ are used in the radial basis function (4). Since all three data sets are normalized between 0 and 1 at the very beginning, we used the same $\sigma$ value for all experiments. Meanwhile, 30 different partitions were utilized in each epoch. Each experiment was run 30 times, since the FCM algorithm has significant sensitivity to cluster center initialization and it is easy to get stuck in local extrema. Another reason is that the result from one trial may vary and may not be enough to tell how many clusters really exist.

TABLE I

Parameter Values

\begin{tabular}{|c|c|}
\hline Parameter & Value \\
\hline Number of ants & 30 Partitions \\
\hline Memory per ant & 5 \\
\hline Iterations per epoch & 50 \\
\hline Epochs & 1000 \\
\hline$P_{\text {rest }}$ & 0.01 \\
\hline$P_{\text {continue }}$ & 0.75 \\
\hline$P_{\text {ContinueCurrent }}$ & 0.20 \\
\hline$D_{\min }$ & 0.001 \\
\hline$D_{\max }$ & 0.01 \\
\hline $\mathrm{m}$ & 2 \\
\hline$\sigma(k e r n e l)$ & 0.1 \\
\hline $\mathrm{a}($ kernel $)$ & 2 \\
\hline $\mathrm{b}(\mathrm{kernel})$ & 1 \\
\hline
\end{tabular}

\section{B. Results}

There is always an argument about the number of classes for the Iris data, and in our previous work [2] two classes were found. Many experiments have shown a nice separation between class 1 and class 2 /class 3 . It is obvious that overlap exists between class 2 and class 3 with the petal length and petal width feature projection shown in Fig. 1. In our experiments, different from in [2], the results clearly indicate that there were three classes (Table II). It was not too surprising to see three clusters found by using the kernelized Fuzzy ants algorithm. The main idea of the kernel method is to transform an original low-dimensional input space into a high-dimensional feature space, therefore, the clusters which overlap in the original feature space may be separated in kernel space.

We used the same threshold strategy as in [2], since the FCM functional has a bias towards producing equal size clusters, we want to use the threshold to eliminate spurious clusters. The threshold equation is

$$
T=\frac{n}{c} * P
$$

where $\mathrm{n}$ is the number of examples, $\mathrm{c}$ is the number of clusters searched for and $\mathrm{P}$ is the desired percentage of the average size. If a cluster had less than the threshold, it meant that there was no cluster and the cluster should be merged with other clusters. When starting the cluster search with three clusters, a partition with 50 examples from cluster 1 was found 29 out of 30 times. An example from this experiment is $(50,45,55)$. The other two clusters still overlapped to some degree. In 7 out of 30 trials there were only two clusters, but the result of the average partition found at $P \geq 20$ still led to the conclusion that there were three clusters in the Iris data when we searched for 


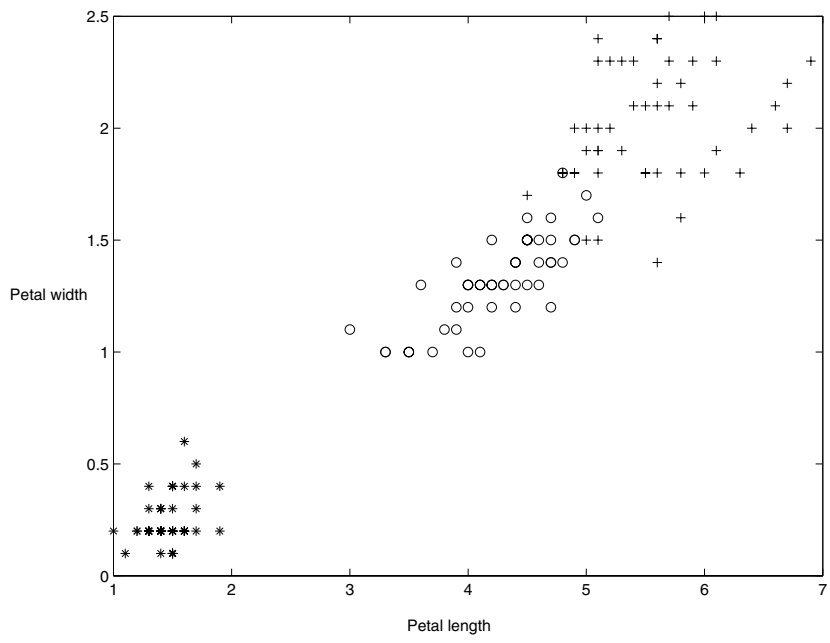

Fig. 1. Iris Dataset - petal length and petal width features

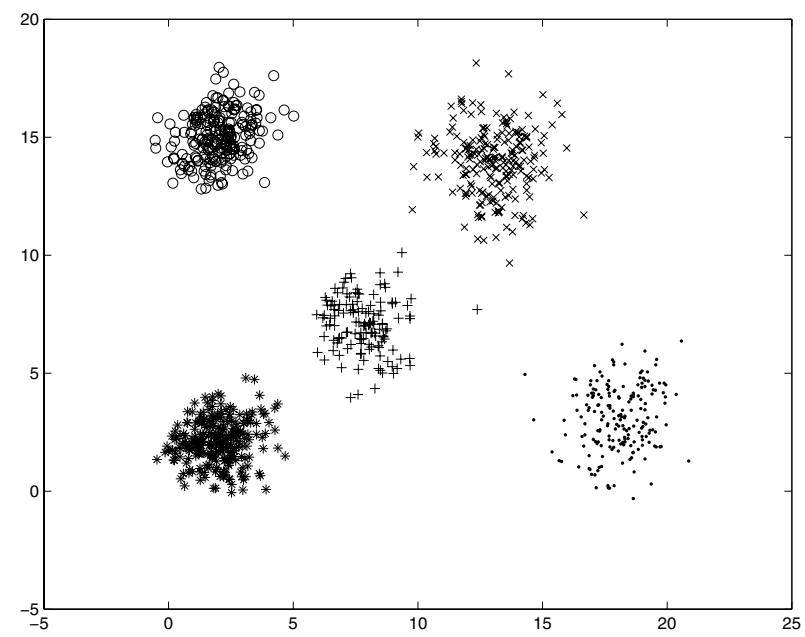

Fig. 2. Gauss Dataset

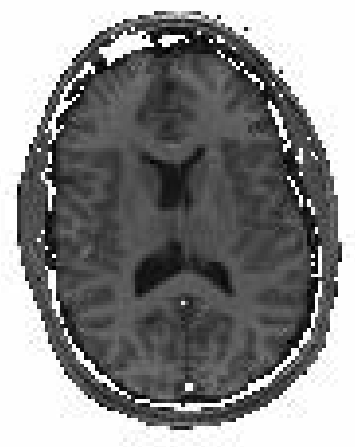

Fig. 3. MRI dataset three. When searching for four clusters in the Iris data, there was always an empty cluster or almost empty cluster (only a few examples inside) in the case that three clusters were found. Many of experiments still had a partition with 50 examples from cluster 1 and the other two clusters overlapped. Interestingly, a perfect partition $(50,50,50,0)$ occurs twice. By looking at Table II, any threshold above 30 percent would indicate that there were three clusters. As for the cases in which five or six clusters were searched for, a number of times only two clusters were found. This did not change the predominant conclusion that three clusters existed in both experiments. However, both answers ( 2 or 3 clusters) seem plausible.

The artificial data is very simple, it doesn't have noise as shown in Fig. 2, so we expected to get good results from this dataset. We did experiments searching for 5, 6, 7, 8 and 9 clusters. Results are summarized in Table III. Surprisingly, the ant based clustering always found five clusters when searching for five and they made up the original partition 30 out of 30 times. This was better than the result in [2], which had a perfect partition 15 out of 30 times. When searching for six clusters, it found five clusters for a $P \geq 30,29$ times. One time it found six clusters, with cluster 5 split into two chunks, but both chunks still existed after thresholding. When it searched for seven, 28 times it found five clusters for $P \geq 30$. The two cases where six clusters were found (248, $133,217,143,210,45,5)$ and $(248,133,217,129,210$, $63,0)$, show that cluster 4 was split into three clusters and two clusters respectively. When searching for eight and nine clusters, five clusters were found 29 times with the higher $P$ values shown in Table III.

The MRI data set, which has 6535 examples after air voxels were removed to speed up the computation time, consists of three clusters as shown Fig. 3. Those three clusters are unequally sized, CSF has fewer voxels than white matter and gray matter. Also, a real problem is we don't have label information for each voxel, hence, we don't know exactly how many voxels belong to each cluster (tissue). Approximately the white matter and gray matter classes have over 2000 voxels, and CSF has over 800 voxels. The choice of $P$ value was based on this information. The summary of results are shown in Table IV, apparently we can conclude that three clusters exist, which is exactly what was expected. When searching for three clusters, exactly three clusters were found for a percentage 30 or greater. A typical partition was $(2547,3038,950)$. In searching for four clusters, three clusters were found 29 out of 30 times, one time two clusters were found. One cluster split into 2 chunks, however those two chunks were thresholded away. A similar situation took place when searching for five or six clusters. The FCM algorithm is really not the right functional to use for widely different sized clusters and the threshold must be carefully set when cluster sizes are unequal. In most experiments three clusters were discovered. 
TABLE II

NUMBER OF CLUSTERS SEARCHED FOR AND AVERAGE NUMBER FOUND

FOR THE IRIS DATA WITH THE MINIMUM P OVER 30 TRIALS.

\begin{tabular}{|c|c|c|}
\hline Clusters searched & Ave. clusters found & P \\
\hline 3 & 2.76 & 0.2 \\
\hline 4 & 3 & 0.3 \\
\hline 5 & 2.8 & 0.9 \\
\hline 6 & 2.8 & 0.9 \\
\hline
\end{tabular}

TABLE III

NUMBER OF CLUSTERS SEARCHED FOR AND AVERAGE NUMBER FOUND

For the ARTIFicial DATA With THE Minimum P OVER 30 TRIALS

\begin{tabular}{|c|c|c|}
\hline Clusters searched & Ave. clusters found & $\mathrm{P}$ \\
\hline 5 & 5 & 0.3 \\
\hline 6 & 5.033 & 0.3 \\
\hline 7 & 5.06 & 0.3 \\
\hline 8 & 5.033 & 0.75 \\
\hline 9 & 5 & 0.8 \\
\hline
\end{tabular}

\section{SUMMARY AND DISCUSSION}

A reformulation of the KFCM objective function to remove the dependence on the membership matrix was developed. This reformulation was integrated into the XieBeni partition validity metric. The Xie-Beni metric was then used as the evaluation function for a swarm based clustering algorithm. Using an ant paradigm, the ants were assigned to move features of cluster centers of partitions in unison. Multiple partitions were explored simultaneously. The advantage of using the partition validity metric was shown to be that the cluster substructure of the data could be discovered. Experiments were done with three data sets, the Iris data, an artificial five class data set and a three Tesla T1 weighted MRI image. A threshold was used to remove small clusters. The results were that three clusters were generally discovered for the three class Iris data, three clusters were discovered for the three class MRI data and the original partition of data was discovered for the artificial data set in many cases (five clusters in general).

The substructure discovery simply depends upon choosing an overestimate of the true number of clusters. This seems to be generally possible. Also, the method was not very

TABLE IV

\section{NUMBER OF CLUSTERS SEARCHED FOR AND AVERAGE} NUMBER FOUND

FOR THE MRI DATA WITH THE MINIMUM P OVER 30 TRIALS.

\begin{tabular}{|c|c|c|}
\hline Clusters searched & Ave. clusters found & P \\
\hline 3 & 3 & 0.3 \\
\hline 4 & 2.97 & 0.5 \\
\hline 5 & 2.97 & 0.6 \\
\hline 6 & 2.97 & 0.7 \\
\hline
\end{tabular}

sensitive to how large the overestimate was. It was effective even when two times the number of clusters was estimated. The results using a kernel based fuzzy clustering approach exceed those reported in [2] with FCM using the Euclidean distance. The final result can be combined into a viable partition (indeed the exact clusters were found in many cases for the artificial data) or the final cluster centers could serve as an excellent initialization for (a few iterations of) FCM.

Several questions deserve future consideration. The first is how to set the threshold (via P) which would indicate whether a cluster is spurious, for different data sets, it seemed the percentage value varied. It depends on the number of clusters and the number of clusters searched for. If the resulting threshold is too large, a cluster split into two or more chunks could be left undiscovered (all sub-clusters are undiscovered). The second question is how to set the $\sigma$ in equation (4). As $\sigma$ becomes greater than 1 or even larger, the value of equation (5) tends to be 0 , which violates our purpose in using kernel function, we want to make the distance between the example and centroids (kernel space) large, not small. We also don't want $\sigma$ too small, because it will make (5) approach a constant number 2 . Therefore, the selection of $\sigma$ value is also very important.

\section{ACKNOWLEDGMENT}

This research was partially supported by the National Institutes of Health under grant number 1 R01 EB00822-01 and by the Department of Energy through the Advanced Strategic Computing Initiative (ASCI) Visual Interactive Environment for Weapons Simulation (VIEWS) Data Discovery Program Contract number: DEAC04-76DO00789.

\section{REFERENCES}

[1] X. Xie and G. Beni, "Validity measure for fuzzy clustering," IEEE Transactions on Pattern Analysis and Machine Intelligence, Vol. 3, no. 8, pp. 841-846, 1991.

[2] Hall, L.O. and Kanade, P.M., "Swarm Based Fuzzy Clustering with partition Validity,' Fuzzy Systems, 2005. FUZZ '05. The 14th IEEE International Conference on May 22-25, 2005 Page(s): 991 - 995.

[3] R. J. Hathway and J. C. Bezdek, "Optimization of clustering criteria by reformulation," IEEE Transactions on Fuzzy System, vol. 3, no. 2, pp. 241-245, May 1995.

[4] Songcan Chen and Daoqiang Zhang, "Robust image segmentation using FCM with spatial constraints based on new kernel-induced distance measure," Systems, Man and Cybernetics, Part B, IEEE Transactions on Volume 34, Issue 4, Aug. 2004 Page(s): 1907 - 1916.

[5] J-H. Chiang, P-Y. Hao, "A new kernel-based fuzzy clustering approach: support vector clustering with cell growing," IEEE Transactions on Fuzzy Systems, Volume 11, Issue 4, Aug. 2003 Page(s):518 - 527.

[6] D-Q Zhang and S-C Chen, "Clustering Incomplete Data Using KernelBased Fuzzy C-means Algorithm," Neural Processing Letters, Volume 18, Issue 3, Dec 2003, Pages 155 - 162.

[7] M. Girolami, "Mercer kernel-based clustering in feature space," IEEE Trans. Neual Networks, vol. 13, pp. 780-784, May 2002.

[8] J.M. Leski, Fuzzy c-varieties/elliptotypes clustering in reproducing kernel Hilbert space Fuzzy Sets and Systems, Volume 141, Number 2, 2004, pp. 259-280.

[9] Mark S. Cohen *, Richard M. DuBois, Michael M. Zeineh, "Rapid and effective correction of RF inhomogeneity for high field magnetic resonance imaging," Human Brain Mapping, Volume 10, Issue 4 , Jul. 2000 Pages $204-211$

[10] P. M. Kanade and L. O. hall, "Fuzzy ants clustering with centroids," FUZZ-IEEE'04, 2004.

[11] P. Kanade, "Fuzzy ants as a clustering concept," Master's thesis, University of South Florida, Tampa, FL 2004 